\title{
Overview of the Clouds and the Earth's Radiant Energy System (CERES) Experiment
}

\author{
Norman G. Loeb and Bruce A. Wielicki \\ NASA Langley Research Center, Mail Stop 420, Hampton, VA \\ n.g.loeb@larc.nasa.gov
}

\begin{abstract}
This paper presents an overview of the CERES project. It demonstrates how algorithm improvements have lead to improved top-of-atmosphere (TOA) radiative flux accuracy. CERES shortwave flux anomalies are compared with those from Earthshine and ISCCP-FD.

OCIS codes: (010.1310) Atmospheric scattering
\end{abstract}

\section{Introduction}

Clouds and the Earth's Radiant Energy System (CERES) investigates the critical role that clouds and aerosols play in modulating the radiative energy flow within the Earth-atmosphere system. CERES builds upon the foundation laid by previous missions, such as the Earth Radiation Budget Experiment (ERBE), to provide highly accurate topof-atmosphere (TOA), within-atmosphere and surface radiative fluxes together with coincident cloud and aerosol properties inferred from high-resolution imager measurements. The CERES instrument is a scanning broadband radiometer that measures filtered radiances in the SW (wavelengths between 0.3-5 $\mu \mathrm{m}$ ), total (TOT) (wavelengths between 0.3-200 $\mu \mathrm{m}$ ) and window (WN) (wavelengths between 8-12 $\mu \mathrm{m}$ ) regions. The spatial resolution of CERES at nadir is $10 \mathrm{~km}$ on the Tropical Rainfall Measuring Mission (TRMM) and $20 \mathrm{~km}$ on the Terra and Aqua spacecrafts. One of the unique features of CERES is its ability to scan in several different modes. Typically, CERES is placed in one of three scan modes: crosstrack, alongtrack, and rotating azimuth plane (RAP). The crosstrack scan is perpendicular to the ground track and optimizes spatial sampling but has limited angular sampling; the alongtrack scan provides measurements near the satellite orbital plane from several near-simultaneous viewing zenith angles over the same region; the RAP scan provides multiangle measurements from a number of viewing zenith and relative azimuth angles by scanning in elevation as it rotates in azimuth. Figure 1 shows the data processing flow of CERES. In order to maintain continuity with the TOA radiation budget from ERBE, one subsystem of CERES produces ERBE-Like products obtained by applying algorithms developed during ERBE to CERES data. The new generation of data products from CERES takes full advantage of coincident imager measurements from the Visible Infrared Radiometer (VIRS) on TRMM and the Moderate Resolution Imaging Spectroradiometer (MODIS) on Terra and Aqua. The imager measurements are used to infer cloud properties [1], such as cloud optical depth, cloud fraction, cloud phase, cloud height, effective radius, etc., as well as aerosol properties [2,3]. The high-resolution cloud and aerosol properties are weighted by the CERES point-spread function to provide a spatially matched cloudaerosol-radiation dataset. The improved scene identification from the imager and the ability of CERES to optimize

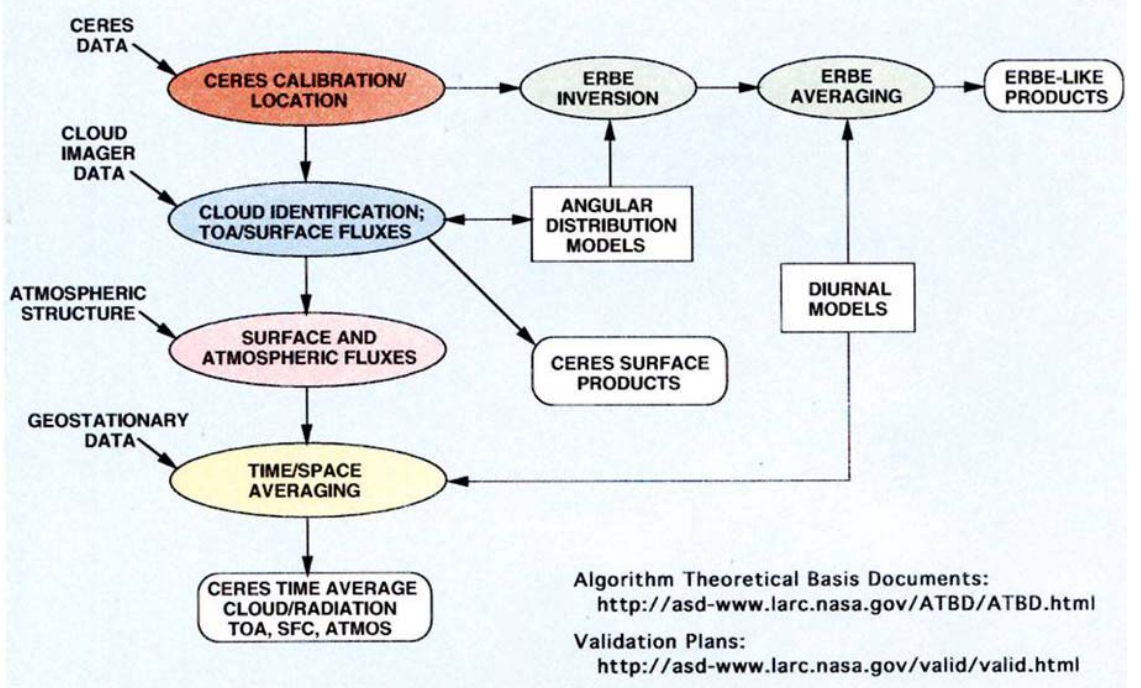

Figure 1 CERES data processing flow. angular sampling of the radiation field through the RAP scan mode was used to produce improved Angular Distribution Models (ADMs) for converting measured radiances to radiative fluxes [4-7]. The imager-based cloud and aerosol properties and meteorological information from the Global Modeling and Assimilation Office (GMAO)'s Goddard Earth Observing System DAS (GEOS-DAS V4.0.3) product [8], are used as input to a radiative transfer model in order to compute radiative fluxes at the surface and at various levels within the atmosphere [9]. Since the satellites that carry CERES instruments sample a given location only a few times per day (e.g., twice per day at the 
equator), 3-hourly geostationary data are used in conjunction with CERES in order to account for the influence of diurnal changes in the clouds and radiation in CERES monthly mean data products [10]. To produce its data products, up to 11 instruments on 7 spacecraft are all integrated to obtain climate accuracy in top to bottom fluxes.

Clearly, it is not possible to summarize all of the exciting new results from CERES in a 3-page report. Therefore, we restrict the discussion to recent results that demonstrate how improvements in CERES ADMs have lead to improved TOA flux accuracy. Validation results include TOA flux self-consistency tests and comparisons with MISR albedos. We also compare global SW TOA flux anomalies with other datasets such as Earthshine and ISCCP.

\section{Results}

A useful consistency test of ADMs is to stratify regional or global mean TOA fluxes by satellite viewing zenith angle (Figs. 2a-d). Since radiative flux is by definition independent of viewing geometry, flux retrievals obtained by applying the ADMs at different angles should not depend on viewing geometry. As shown in Fig. 2, albedos based on the ERBE ADMs systematically increase by $4 \%$ and $8 \%$ with viewing zenith angle in January and July 2003, respectively. In contrast, albedos inferred from the CERES Terra ADMs show a smaller increase of 1\%-2\%. ERBE-
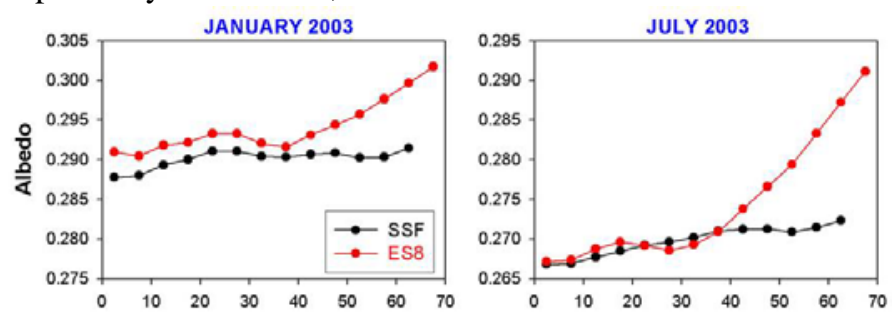

like LW TOA fluxes show a systematic decrease with viewing zenith angle of $2 \%-2.4 \%$, whereas fluxes from the CERES Terra ADMs remain within $0.7 \%-0.8 \%$ at all angles.

Figure 3 presents the first direct comparison of CERES and MISR albedos based on an analysis by [11]. In order to compare CERES and MISR albedos, an algorithm for converting

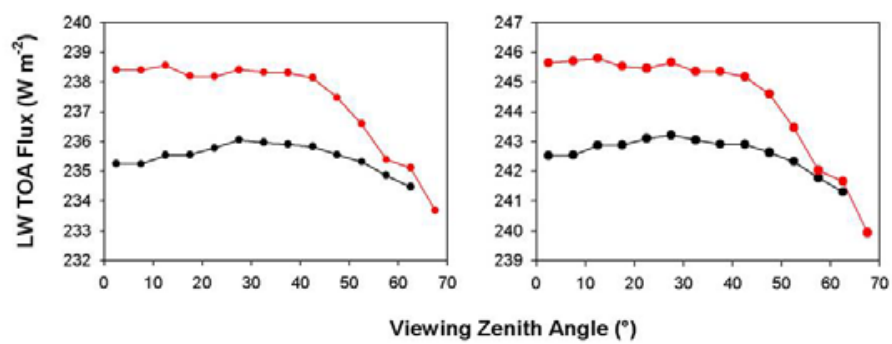

Figure 2 SSF and ES8 global albedo against viewing zenith angle for (a) January and (b) July 2003. LW TOA flux against viewing zenith angle for (c) January and (d) July 2003. MISR spectral albedos to broadband albedos is derived using a new merged CERES-MISRMODIS dataset [12]. For overcast $1^{\circ} \times 1^{\circ}$ ocean regions, the relative differences and the relative root-mean-square (RMS) differences between MISR and CERES albedos are $\sim 0.81 \%$ and $\sim 4.29 \%$, respectively. Since there is a $\sim 2 \%$ error due to the narrow-to-broadband albedo conversion, the RMS differences between MISR and CERES albedos due to ADM differences alone is estimated to be smaller than $\sim 3.8 \%$.

Recently a number of publications have emerged showing contradictory changes in planetary albedo during the first part of the 21st century. Using ground-based measurements of Earthshine at the Big Bear Solar Observatory (BBSO) in California, [13], find that the Earth's reflectance has increased by as much as 0.018 between 1999 and 2003, corresponding to a change of $\sim 6 \mathrm{Wm}^{-2}$ in TOA radiative flux. This approach estimates the Earth's albedo using telescope measurements of the visible solar radiation that is first reflected by the
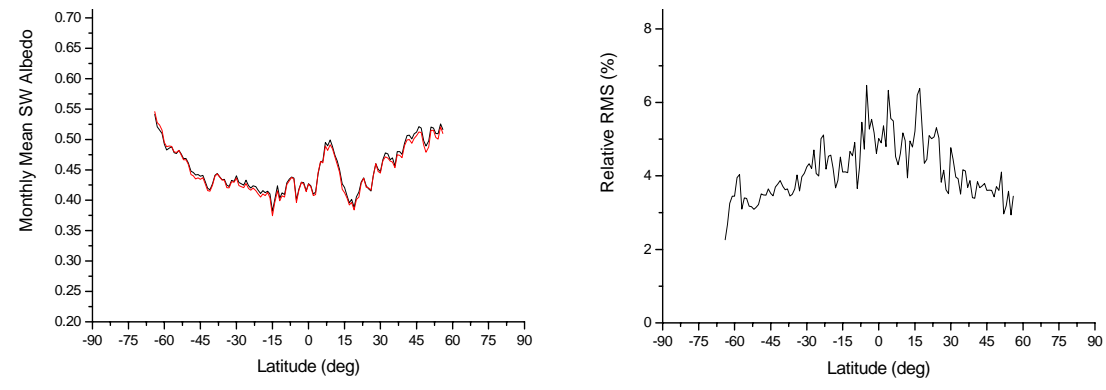

Figure 3 (a) The monthly zonal mean of the MISR (black) and CERES (red) albedos for overcast ocean scenes in October 2001; (b) the monthly zonal mean of the relative RMS differences between the instantaneous $10 \mathrm{x} 10-$ region-averaged MISR and CERES albedos.
Earth towards the Moon and then back from the Moon to an observer on the night-side of the Earth. The BBSO Earthshine measurements sample only $\sim 1 / 3$ of the Earth on any given day, and cover at most $\sim 1 / 3$ of each lunar month provided the night-sky is cloud-free. A 6 $\mathrm{Wm}^{-2}$ change is climatologically significant as it exceeds the radiative forcing caused by Mount Pinatubo in the early 1990s by a factor of 2.5, and is twice as large as the longwave radiative forcing by greenhouse gases since 1850 . In 


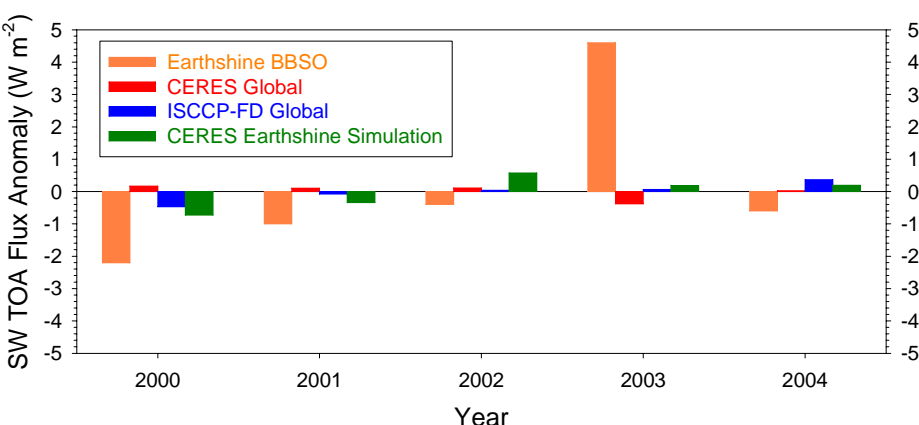

Figure 4 Global annual mean SW TOA flux anomalies from Earthshine BBSO, CERES Terra global fluxes, ISCCP-FD, and a simulation of Earthshine. contrast, the global CERES observations show a very different result, as illustrated in Figure 4. In fact, none of the other methods considered (including ISCCP-FD, [14]) support the dramatic increase in reflectance obtained by BBSO Earthshine between 2000 and 2003. The standard deviation in the BBSO Earthshine anomalies is a factor of 5 greater than simulated Earthshine anomalies (using CERES data), and over an order-ofmagnitude greater than those from CERES global results. Interestingly, anomalies based on the simulated CERES Earthshine approach bear little resemblance to the CERES global results. In fact, the standard

deviation in the simulated CERES Earthshine anomalies is a factor of 2 larger than the CERES global results. While ISCCP and CERES SW TOA flux anomalies also differ from one another from year to year, the standard deviation in the anomalies is within $40 \%$ of each other.

\section{Conclusions}

The CERES project provides cloud-aerosol-radiation data products for studying the influence of clouds and aerosols on the Earth's radiation budget. CERES goes well beyond previous radiation budget projects (e.g., ERBE) by merging broadband CERES radiative fluxes with coincident imager-based cloud and aerosol properties. The new algorithms created during CERES have lead to improved accuracy in TOA fluxes by a factor of 2-5 compared to ERBE. The additional imager data allow accurate estimates of radiative flux profiles within the atmosphere and at the surface. Merging CERES with geostationary data leads to improved characterization of diurnal variations in clouds and radiation which in turn leads to improved monthly mean radiative fluxes and cloud properties. Global shortwave radiative flux anomalies from CERES during the first 5 years of the $21^{\text {st }}$ century are in stark contrast to those derived from ground-based measurements of Earthshine. The CERES data do not support the $6 \mathrm{Wm}^{-2}$ increase in reflected solar radiation obtained from Earthshine.

\section{References}

[1] P. Minnis, et al., "CERES Cloud Property Retrievals from Imagers on TRMM, Terra, and Aqua" Proc. SPIE 10th International Symposium on Remote Sensing: Conference on Remote Sensing of Clouds and the Atmosphere VII, Barcelona, Spain, September 8-12, 2003, 37-48.

[2] L.A. Remer, et al., The MODIS Aerosol Algorithm, Products and Validation, J. Atmos. Sci., 62, 947-973 (2005).

[3] A. Ignatov and L. L. Stowe, Aerosol retrievals from individual AVHRR channels: I. Retrieval algorithm and transition from Dave to 6S radiative transfer model. J. Atmos. Sci., 59, 313-334 (2002).

[4] N.G. Loeb, et al., Angular distribution models for top-of-atmosphere radiative flux estimation from the Clouds and the Earth's Radiant Energy System instrument on the Tropical Rainfall Measuring Mission Satellite. Part I: Methodology, J. Appl. Meteor., 42, 240-265 (2003).

[5] N.G. Loeb, et al., Angular distribution models for top-of-atmosphere radiative flux estimation from the Clouds and the Earth's Radiant Energy System instrument on the Tropical Rainfall Measuring Mission Satellite. Part II: Validation, J. Appl. Meteor., 42, 1,748-1,769 (2003).

[6] N.G. Loeb, et al., Angular distribution models for top-of-atmosphere radiative flux estimation from the Clouds and the Earth's Radiant Energy System instrument on the Terra Satellite. Part I: Methodology. J. Atmos. Ocean. Tech., 22, 338-351 (2005).

[6] N.G. Loeb, et al., Angular distribution models for top-of-atmosphere radiative flux estimation from the Clouds and the Earth's Radiant Energy System instrument on the Terra Satellite. Part II: Validation. J. Atmos. Ocean. Tech., (in press) (2005).

[8] M.J. Suarez, Technical report series on global modeling and data assimilation, Vol. 26: Documentation and validation of the Goddard Earth Observing System (GEOS) data assimilation system—Version 4, NASA/TM-2005—104606, Vol. 26, 181 pp., Washington, DC. (2005)

[9] T.P. Charlock, et al., Compute Surface and Atmospheric Fluxes (System 5.0), CERES Algorithm Theoretical Basis Document. 84 pp., [Available from http://asd-www.larc.nasa.gov/ATBD/pdf_docs/r2_2/ceres-atbd2.2-s5.0.pdf] (1997).

[10] D.F. Young, et al., Temporal interpolation methods for the Clouds and the Earth’s Radiant Energy System (CERES) experiment. J. Appl. Meteor., 37, 572-590 (1998).

[11] W. Sun, et al., Comparison of MISR and CERES Top-of-Atmosphere Albedo, Geophys. Res. Lett., submitted (2006).

[12] N.G. Loeb, et al., Fusion of CERES, MISR and MODIS measurements for top-of-atmosphere radiative flux validation, J. Geophy. Res., in press (2006)

[13] E. Pallé, Changes in the Earth’s reflectance over the past two decades, Science, 304, 1299-1301, doi:10.1126/science.1094070 (2004).

[14] Y. Zhang, et al., Calculation of radiative fluxes from the surface to top of atmosphere based on ISCCP and other global data sets: Refinements of the radiative transfer model and the input data, J. Geophys. Res., 109, D19105, doi:10.1029/2003JD004457 (2004).

[15] N.G. Loeb, et al., Variability in Global Top-of-Atmosphere Shortwave Radiation between 2000 and 2005, Geophys. Res. Lett, submitted (2006). 